ARTICLE

\title{
Axotomy-induced HIF-serotonin signalling axis promotes axon regeneration in C. elegans
}

Tanimul Alam ${ }^{1, \star}$, Hiroki Maruyama ${ }^{1, \star}$, Chun Li$^{1}$, Strahil Iv. Pastuhov ${ }^{1}$, Paola Nix ${ }^{2}$, Michael Bastiani ${ }^{2}$, Naoki Hisamoto ${ }^{1} \&$ Kunihiro Matsumoto ${ }^{1}$

The molecular mechanisms underlying the ability of axons to regenerate after injury remain poorly understood. Here we show that in Caenorhabditis elegans, axotomy induces ectopic expression of serotonin (5-HT) in axotomized non-serotonergic neurons via HIF-1, a hypoxia-inducible transcription factor, and that $5-\mathrm{HT}$ subsequently promotes axon regeneration by autocrine signalling through the SER-7 5-HT receptor. Furthermore, we identify the rhgf-1 and rga-5 genes, encoding homologues of RhoGEF and RhoGAP, respectively, as regulators of axon regeneration. We demonstrate that one pathway initiated by SER-7 acts upstream of the C. elegans RhoA homolog RHO-1 in neuron regeneration, which functions via G12 $\alpha$ and RHGF-1. In this pathway, RHO-1 inhibits diacylglycerol kinase, resulting in an increase in diacylglycerol. SER-7 also promotes axon regeneration by activating the cyclic AMP (cAMP) signalling pathway. Thus, HIF-1-mediated activation of 5-HT signalling promotes axon regeneration by activating both the RhoA and cAMP pathways.

\footnotetext{
${ }^{1}$ Division of Biological Science, Graduate School of Science, Nagoya University, Chikusa-ku, Nagoya 464-8602, Japan. ${ }^{2}$ Department of Biology, University of Utah, 257 South 1400 East, Salt Lake City, Utah 84112-0840, USA. * These authors contributed equally to this work. Correspondence and requests for materials should be addressed to N.H. (email: i45556a@cc.nagoya-u.ac.jp) or to K.M. (email: g44177a@nucc.cc.nagoya-u.ac.jp).
} 
A $\mathrm{n}$ axon's ability to regenerate after injury depends on both its intrinsic growth capacity and the external environment. Most invertebrate neurons, as well as neurons in the mammalian peripheral nervous system are able to regenerate. Although intrinsic regeneration signals can influence regenerative success, the variables determining a neuron's intrinsic regrowth capacity remain poorly defined at the molecular level. The nematode Caenorhabditis elegans has recently emerged as a tractable genetic model for studying neuron regenerative processes $^{1,2}$. Two recent screens have identified candidate genes that may be involved in axon regeneration: Chen et al. ${ }^{3}$ screened a collection of mutants of $C$. elegans, and Nix et al. ${ }^{4}$ screened using an RNA interference (RNAi) library. The candidate genes identified include those encoding both growth-promoting and growth-inhibiting factors. Several of these genes have been implicated previously in regeneration and others define new and conserved pathways of interest.

In the present study, we investigate the roles of the tph-1 and $\mathrm{rga}$ 5 genes in the regulation of axon regeneration. The tph- 1 and $r g a-5$ genes were identified as candidate genes in a screen of mutants and by an RNAi screen, respectively ${ }^{3,4}$. The $t p h-1$ gene encodes an enzyme involved in 5 -HT biosynthesis ${ }^{5}$, while the rga-5 gene encodes a homolog of the mammalian p190 Rho GTPase-activating protein. We show that the hypoxia-inducible transcription factor (HIF-1) induces expression of the tph-1 gene in axotomized neurons, which are not serotonergic. 5-HT subsequently promotes axon regeneration by signalling through the serotonin receptor SER-7. We further demonstrate that the RhoA signalling pathway is important for neuronal regeneration via its ability to inhibit diacylglycerol (DAG) kinase, thus increasing DAG concentrations. These two effects are linked, as C. elegans RhoA is activated by SER-7 and by G12 $\alpha$ through RhoGEF encoded by the rhgf- 1 gene. SER-7 is also required for axon regeneration via its ability to activate the cyclic AMP (cAMP) signalling pathway. Thus, HIF-1mediated activation of 5-HT synthesis and consequent autocrine signalling promotes the neuron regenerative response by activating two separate signalling pathways.

\section{Results}

5-HT is required for axon regeneration. It has been suggested that axon injury may represent a type of cellular stress. In response to specific types of stress, neurotransmitters are deployed to effect the appropriate physiological and behavioural modifications ${ }^{6-8}$. In C. elegans, 5 -HT modulates a variety of stress responses $^{9,10}$. However, little is known about how specific types of stress induce 5-HT-mediated signal transduction in the nervous system. Interestingly, Chen et al. ${ }^{3}$ recently carried out a systematic screen of mutants looking for defects in axon regeneration and identified tph-1 among several genes that positively regulate axon regeneration in a glutaminergic touch sensory neuron ${ }^{3}$. The tph-1 gene encodes tryptophan hydroxylase ${ }^{5}$, which is required for 5-HT biosynthesis (Fig. 1a). We confirmed that a loss-of-function mutation $(m g 280)$ of $t p h-1$ caused a defect in axon regeneration in touch sensory posterior lateral microtubule (PLM) neurons (Fig. 1b,c and Supplementary Table 1). Furthermore, the mg280 and $n 4622$ mutations of $t p h-1$ were also defective in axon regeneration in GABA-releasing D-type motor neurons (Fig. 1d and Supplementary Table 2). Exogenous 5-HT treatment restored axon regeneration in tph-1 mutants. These results suggest that $5-\mathrm{HT}$ is generally required during axon regeneration.

We next searched for the 5-HT-producing neurons essential for axon regeneration. To match serotonin levels to the normal range for each neuron, we used a system recently developed by Flavell et al. ${ }^{11}$ A single floxed copy of the $t p h-1$ gene, including the promoter, exons and introns, was inserted into a defined location on chromosome IV (Fig. 1e). This inserted gene was able to rescue the $t p h-1$ defect in axon regeneration of D-type motor neurons (Fig. If and Supplementary Table 2). This was combined with cell-specific transgenes expressing Cre recombinase in amphid sensory neuron, dual cilia, designation F. (ADF), neurosecretory motor neuron (NSM) or hermaphrodite specific neuron (HSN) neurons, which are known to be 5-HT-producing neurons ${ }^{5}$, or in D-type neurons. This system allowed the excision of the inserted $t p h-1$ gene and elimination of 5-HT production in these individual neurons. We found that the $t p h-1$ defect was still rescued when Cre was expressed in ADF, NSM or HSN neurons (Fig. If and Supplementary Table 2). In contrast, the tph-1 defect was not rescued when Cre was expressed in D-type motor neurons. Furthermore, expression of $t p h-1$ in ADF neurons by the $s r h-142$ promoter was unable to rescue the $t p h-1$ defect (Fig. 1d and Supplementary Table 2). These results suggest that axon regeneration in D-type motor neurons requires 5-HT production by $\mathrm{D}$ neurons. To confirm this, we ectopically expressed $t p h-1$ in D-type motor neurons in a $t p h-1(m g 280)$ background using the unc-25 promoter. We found that expression of tph-1 in $\mathrm{D}$ neurons did indeed suppress the defect in axon regeneration of tph-1 mutants (Fig. 1d and Supplementary Table 2). These results demonstrate that TPH-1 acts to promote regeneration of the damaged neuron in a cell-autonomous manner.

HIF-1 upregulates 5-HT expression in injured neurons. The identification of D-type neurons as the 5-HT-producing neurons essential for axon regeneration was unexpected, as D neurons are known to be GABAergic and the expression of 5-HT synthesis enzymes in these neurons has not been found ${ }^{5,12-14}$. We therefore addressed the possibility that axon severing might induce transcription of the $t p h-1$ gene in D-type neurons. To test this, we monitored expression of the Ptph-1::gfp transgene, which consists of the $t p h-1$ promoter driving the fluorescent protein green fluorescent protein (GFP). We found that expression of $t p h-1$ was altered in D-type neurons by axon injury. In control animals, Ptph$1:: g f p$ was robustly expressed in the head of animals in the NSM and ADF neurons (Supplementary Fig. 1), and was not expressed in D neurons. However, after laser surgery of D-type neurons, Ptph$1:: g f p$ expression was strongly upregulated in these neurons (Fig. 2a,b). This expression became visible at $30 \mathrm{~min}$ after surgery, and returned to undetectable levels by $24 \mathrm{~h}$. Thus, axon injury induces the transient expression of Ptph-1::gfp in severed D-type neurons. 5-HT immunofluorescence staining of animals treated with laser surgery also revealed induction of 5-HT expression, compared with control animals (Supplementary Fig. 2). Expression of Punc-47::mcherry, which reflects the expression of vesicular GABA transporter, was constant over this time period (Fig. 2a). Therefore, the severed GABAergic neurons still keep the GABAergic characteristics, and do not transdifferentiate into serotonergic, non-GABAergic neurons. Furthermore, axon severing also induced 5-HT and Ptph-1::gfp expression in PLM neurons (Supplementary Figs 2 and 3), which are glutamatergic, non-serotonergic touch sensory neurons ${ }^{15}$. Our results demonstrate that axotomized 'non-serotonergic' neurons temporarily gain the ability to synthesize serotonin in response to axon injury.

What determines expression of the ectopic tph-1 gene in response to axon injury? Interestingly, the promoter region of the tph-1 gene contains hypoxia response elements, which are binding sites for HIF-1 (ref. 16). The HIF-1 protein is a transcription factor containing a bHLH-PAS domain that mediates transcriptional responses to hypoxic stress ${ }^{17}$. Indeed, it has been demonstrated that HIF-1 binds to the $t p h-1$ promoter via hypoxia response elements to drive serotonin production 
a

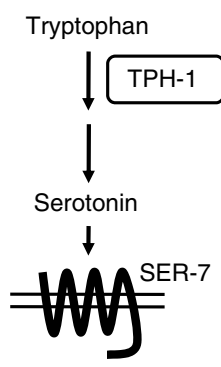

C

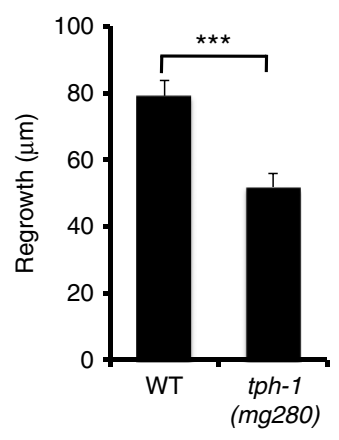

e

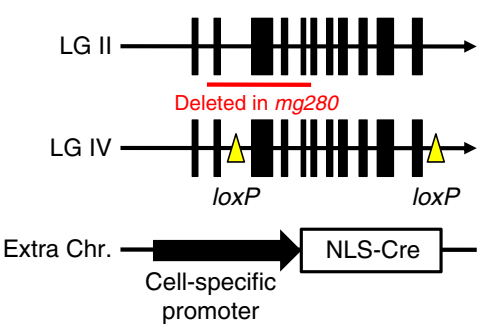

b

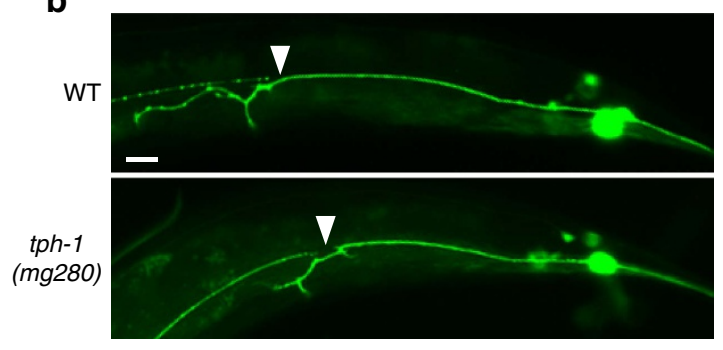

d

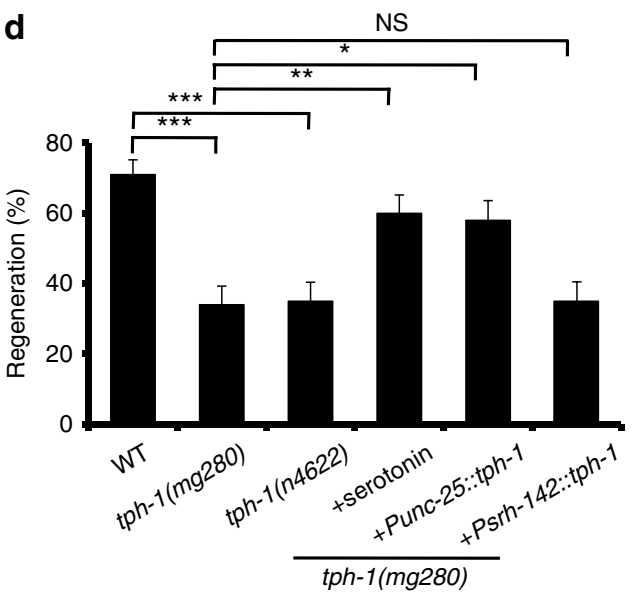

$\mathbf{f}$

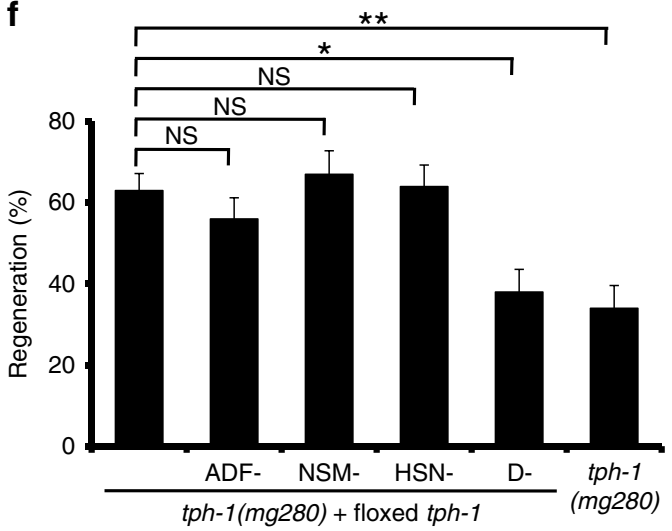

Figure 1 | Serotonin activates axon regeneration. (a) The synthesis of serotonin from tryptophan catalyzed by TPH-1. (b) Representative PLM sensory neurons in wild type and tph-1 mutant animals $24 \mathrm{~h}$ after laser surgery. Arrowheads indicate cut sites. Scale bar $=20 \mu \mathrm{m}$. (c) Regeneration of PLM sensory neurons. Lengths of PLM regrowth at $24 \mathrm{~h}$ after laser surgery are shown. Error bars indicate s.e.m. ${ }^{\star \star \star} P<0.001$ ( $n \geq 30$; unpaired $t$-test, two-tailed and unequal variances). (d,f) Regeneration of D-type motor neurons. Percentages of axons that initiated regeneration $24 \mathrm{~h}$ after laser surgery are shown. Error bars indicate $95 \% \mathrm{Cl}$. ${ }^{\star \star \star} P<0.001 ;{ }^{\star \star} P<0.01$; ${ }^{\star} P<0.05$; NS, not significant (Fisher's exact test, two-tailed, $n \geq 50$ ). (e) Cell-specific deletion of tph-1 using a Cre-loxP recombination system. $\mathrm{Cl}$, confidence interval.

under hypoxic conditions in C. elegans ${ }^{16}$. We therefore examined whether HIF-1 is involved in $t p h-1$ expression in response to axon injury. The hif-1(ia4) mutation had no effect on expression of Ptph-1::gfp in the head of animals (Supplementary Fig. 1) but decreased its induction in response to laser surgery in D-type neurons (Fig. 2a,b), indicating that induction is dependent on HIF-1 function. In addition, expression of Ptph-1::gfp in PLM neurons following laser surgery decreased in hif-1 mutants (Supplementary Fig. 3). Thus, HIF-1 is required for induction of 5-HT expression in injured neurons in response to axon injury.

These results raised the possibility that hypoxic stress might enhance axon regeneration. In normoxic conditions, HIF-1 is degraded through the E3 ubiquitin ligase VHL-1 (von Hippel-Lindau tumour suppressor protein)-dependent degradation pathway ${ }^{18}$. Degradation is initiated through hydroxylation of a specific proline residue in HIF-1 by the oxygen-dependent prolyl 4-hydroxylase EGL-9 (ref. 18). VHL-1 recognizes the hydroxylated proline and targets HIF-1 for ubiquitin-mediated proteasomal degradation ${ }^{19}$. Hypoxic conditions inhibit the hydroxylation of HIF-1, stabilizing the protein and allowing it to transcriptionally regulate expression of its target genes ${ }^{19,20}$. Thus, stabilization of HIF-1 through removal of the HIF-1 degradatory pathway phenocopies the effects of hypoxia seen in neurons. We examined the effect of HIF-1 stabilization on tph-1 expression in D-type motor neurons. For this purpose, we used vhl-1(ok161) mutants and a mutated form of HIF-1, HIF-1(P621A), in which the proline in the conserved LXXLAP motif required for degradation of HIF-1 has been altered to an alanine ${ }^{18,21}$. As observed previously ${ }^{16}$, the $v h l-1$ mutation or expression of HIF-1(P621A) caused constitutive expression of tph-1 in glutamatergic sensory amphid sensory neuron, single cilia, designation G. (ASG) neurons under 
a
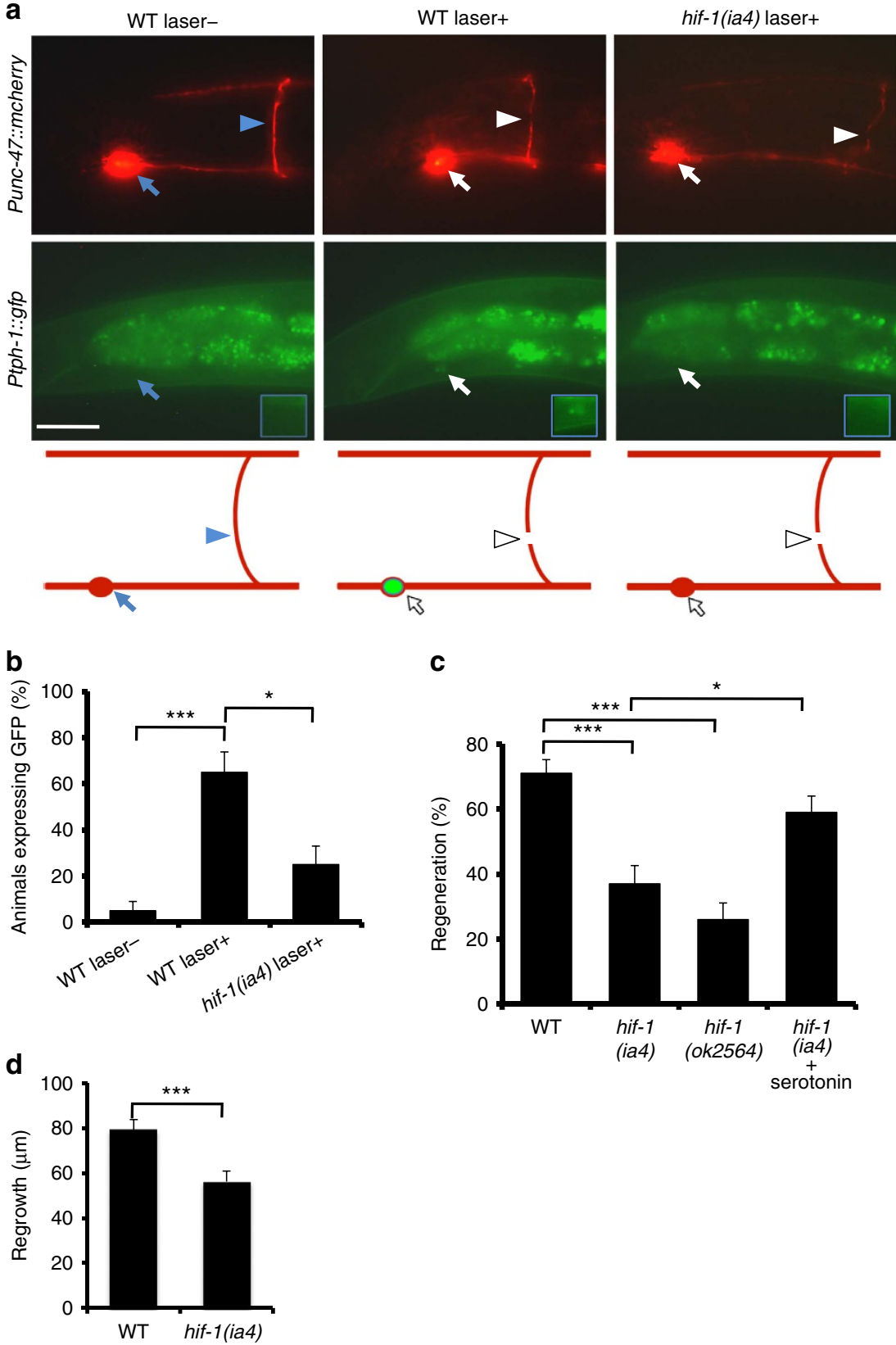

Figure 2 | HIF-1 positively regulates axon regeneration. (a) Induction of Ptph-1::gfp expression in D-type motor neurons by laser surgery. Expression of fluorescent proteins in D-type motor neurons $30 \mathrm{~min}$ after laser surgery is shown. A schematic representation of D-type motor neurons is shown on the bottom. Arrowheads and arrows indicate axons and cell bodies of D-type neurons with (white) or without (blue) laser surgery, respectively. D-type neurons are visualized by $\mathrm{mCherry}$ under control of the unc- 47 promoter. Cell bodies of $\mathrm{D}$-type neurons are magnified (blue boxes). Scale bar $=20 \mu \mathrm{m}$. (b) Percentages of animals expressing tph-1. Induction of Ptph-1::gfp expression in D-type motor neurons with $(+)$ or without $(-)$ laser surgery was assayed as described in Methods section. Twenty neurons were examined for each condition. Error bars indicate $95 \% \mathrm{Cl}$. ${ }^{\star \star \star} P<0.001$; ${ }^{\star} P<0.05$ (Fisher's exact test, two-tailed, $n=20$ ). (c) Regeneration of D-type motor neurons. Percentages of axons that initiated regeneration $24 \mathrm{~h}$ after laser surgery are shown. Error bars indicate $95 \% \mathrm{Cl} .{ }^{\star \star \star} P<0.001 ;{ }^{\star} P<0.05$ (Fisher's exact test, two-tailed, $n \geq 50$ ). (d) Regeneration of PLM sensory motor neurons. Lengths of PLM regrowth at $24 \mathrm{~h}$ after laser surgery are shown. Error bars indicate s.e.m. ${ }^{\star \star \star} P<0.001(n \geq 30$; unpaired $t$-test, two-tailed, unequal variances). $\mathrm{Cl}$, confidence interval.

normoxic conditions (Supplementary Figs 1 and 4). Normally, tph-1 is only weakly expressed under normoxic conditions in these neurons. However, we found that the vhl-1 mutation or expression of HIF-1(P621A) failed to induce Ptph$1:: g f p$ expression constitutively in $\mathrm{D}$ neurons (Supplementary Fig. 4). These results suggest that the mechanism that activates HIF-1 in response to axon injury is different from the HIF-1-stabilizing mechanism that is involved in the response to hypoxia.
Next, we examined whether HIF-1 is required for 5-HT-driven axon regeneration. We found that the frequency of axon regeneration in $\mathrm{D}$ neurons was reduced significantly, in hif-1(ia4) and hif-1(ok2564) mutants, and was restored by exogenous 5-HT treatment (Fig. 2c and Supplementary Table 2). This is consistent with the possibility that HIF-1 regulates axon regeneration by driving $5-\mathrm{HT}$ production. Furthermore, the hif-1(ia4) mutation was also defective in axon regeneration in PLM neurons following laser surgery (Fig. $2 \mathrm{~d}$ and 
Supplementary Table 1). These results suggest that HIF-1 is generally required during axon regeneration.

SER-7 activates axon regeneration via GPA-12 and RHGF-1. The biological effects of 5-HT are mediated through its interactions with $G$ protein-coupled receptors ${ }^{22}$. Since SER-7 is one of the 5-HT receptors, we examined whether SER-7 participates in axon regeneration. We found that two mutations (tm1325 and $t m 1548)$ in the ser-7 gene caused defects in axon regeneration (Fig. 3a and Supplementary Table 2). In contrast to ser-7, the ok345 null mutation of ser-1 encoding another serotonin receptor had no effect on axon regeneration. Thus,
SER-7 plays a specific role in axon regeneration. To test whether SER-7 can act in a cell-autonomous manner, we expressed ser-7 from the $u n c-25$ or $m e c-7$ promoters in $\operatorname{ser}-7(t m 1325)$ mutants. The ser- 7 defect in axon regeneration was rescued by expression of ser-7 in D-type motor neurons by the unc- 25 promoter but not by expression in sensory neurons by the mec-7 promoter (Fig. 3a and Supplementary Table 2). These results demonstrate that SER7 acts to promote regeneration in a cell-autonomous manner in the damaged neuron. Exogenous 5-HT treatment was unable to efficiently suppress the ser-7 defect (Fig. 3a and Supplementary Table 2).

What functions downstream of SER-7 to regulate axon regeneration? SER-7 is coupled to GPA-12, a C. elegans

a
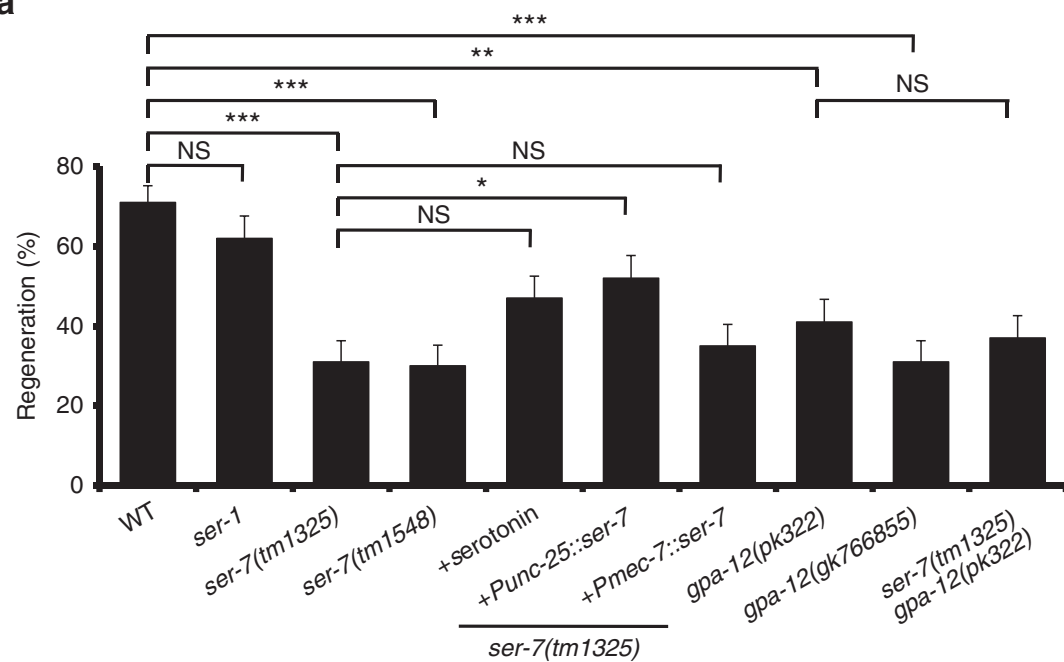

b

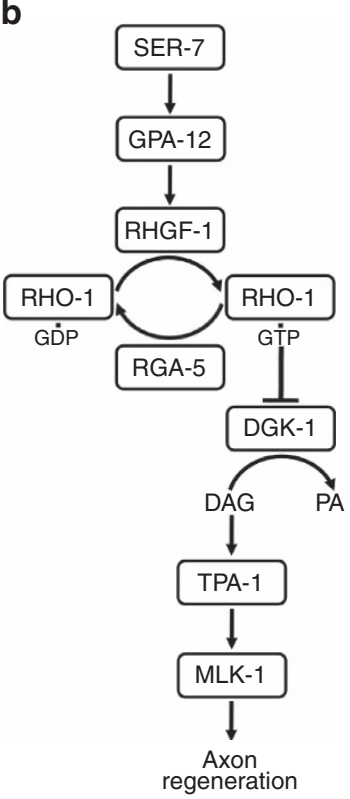

C

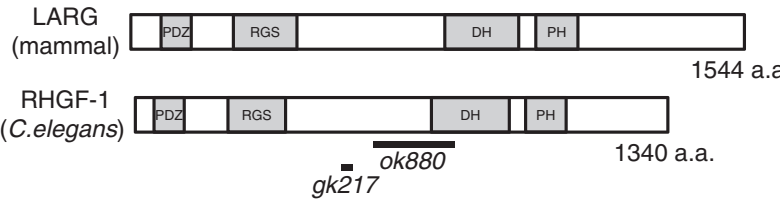

d

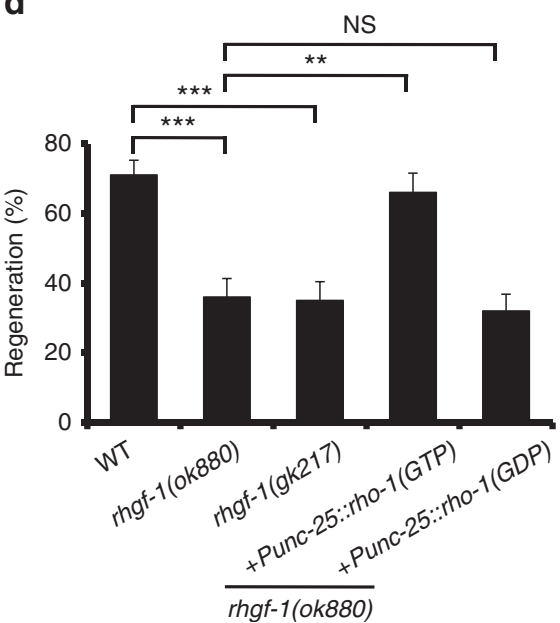

Figure 3 | 5-HT-SER-7 signalling activates axon regeneration via GPA-12 and RHGF-1. (a,d) Percentages of axons that initiated regeneration $24 \mathrm{~h}$ after laser surgery. Error bars indicate $95 \% \mathrm{Cl}$. ${ }^{\star \star \star} P<0.001$; ${ }^{\star \star} P<0.01$; ${ }^{\star} P<0.05$; NS, not significant (Fisher's exact test, two-tailed, $n \geq 50$ ). (b) SER-7 signalling pathway. SER-7 activates the GPA-12-RHGF-1-RHO-1 pathway. Activation of RHO-1 inhibits DGK-1, leading to a stabilization of DAG levels. (c) Structure of RHGF-1. Schematic diagrams of RHGF-1 and its human counterpart are shown. Domains are shown as follows: a PDZ domain, a regulator of G protein signalling domain (RGS), a Dbl homology domain (DH) and a Pleckstrin homology domain (PH). The bold lines underneath indicate the extent of the deleted regions in the rhgf-1(ok880) and rhgf-1(gk217) mutants. 
homologue of the $\alpha$ subunit of the heterotrimeric G12 protein (Fig. 3b) ${ }^{23}$. We found that the $g p a-12(p k 322)$ and $g p a-12(g k 766855)$ mutations significantly reduced axon regeneration after axon injury (Fig. $3 \mathrm{a}$ and Supplementary Table 2). To further address whether ser-7 and gpa-12 function in the same pathway, we constructed ser-7(tm1325); $g p a-12$ (pk322) double mutants. We found that the $g p a-12$ mutation did not enhance the axon regeneration defect of ser-7 mutants (Fig. 3a and Supplementary Table 2), suggesting that SER-7 and GPA-12 function in the same pathway.

In mammals, the RhoGEF LARG protein specifically interacts with $\mathrm{G} 12 \alpha$ via its regulator of $G$ protein signalling (RGS) domain $^{24}$. C. elegans RHGF-1, a homolog of mammalian LARG (Fig. 3c), binds to GPA-12 and acts as a regulator of neurotransmitter release at a subset of cholinergic motor synapses $^{25,26}$. These observations raised the possibility that RHGF-1 may act downstream of GPA-12 in the axon regeneration pathway (Fig. 3b). Indeed, we found that the rhgf-1(ok880) and rhgf-1(gk217) mutants were defective in axon regeneration (Fig. 3d and Supplementary Table 2). Taken together, these results suggest that SER-7 activates axon regeneration through GPA-12 and RHGF-1.

RHO-1 activates axon regeneration by inhibiting DGK-1. The activity of Rho proteins is modulated by their cycling between the active GTP- and inactive GDP-bound states. Rho activity is further regulated by other proteins, including GAPs, which enhance GTP hydrolysis and the consequent inactivation of Rho, and guanine nucleotide exchange factors (GEFs), which promote the binding of GTP and, thus, activation of $\mathrm{Rho}^{27}$. RHO-1 also cycles between the GTP-bound active and the GDP-bound inactive forms. To confirm that RHGF-1 acts as a GEF for RHO-1 and to test if it thereby controls axon regeneration, we expressed a mutant RHO-1 that is locked in the GTP-bound state, RHO-1(G14V), in D-type motor neurons using the unc-25 promoter. We found that expression of RHO-1(G14V) was able to suppress the $r h g f-1$ defect in axon regeneration, whereas a GDP-bound RHO-1(T19N) expressed from the unc-25 promoter was not (Fig. 3d and Supplementary Table 2).

What functions as a RhoGAP in the regulation of axon regeneration? One of the genes identified in our previous RNAi screen in $\beta$-spectrin mutant animals (unc-70 ; ref. 28 ) was rga-5, the product of which belongs to the Rho GTPase-activating protein (GAP) family ${ }^{4}$. RGA-5 has an N-terminal GTP binding domain (GBD) and a C-terminal GAP domain. RGA-5 displays strong homology across its GBD and GAP domains to the mammalian p190 RhoGAP (Fig. 4a and Supplementary Fig. 5). Since the GAP domain in p190 RhoGAP is specific for the Rho family of GTPases, particularly RhoA (ref. 29), we expected that RGA-5 would specifically act on RHO-1. To test this prediction, we performed yeast two-hybrid binding assays to test for interactions between RGA-5 and two RHO-1 variants that are locked in the GTP- and GDP-bound states (G14V and T19N, respectively). We found that the RGA-5 GAP domain (1118-1341 amino acids) specifically interacted with the GTP bound, but not GDP-bound version of RHO-1 (Fig. 4b), consistent with the property of GAP proteins to associate with the GTP-bound version of GTPases. The RGA-5 GAP domain failed to bind to GTP-bound CDC-42 or CED-10, two other Rho/Rac family members, demonstrating that the RGA-5-RHO-1 interaction is specific.

Next, we determined the function of RGA-5 in axon regeneration. First, we examined the rga-5(ok2241) mutation, which deletes the GAP domain in RGA-5 (Fig. 4a). After laser surgery, axons in rga-5(ok2241) mutant animals regenerated normally. However, overexpression of the RGA-5 GAP domain reduced regeneration (Fig. 4c,d and Supplementary Table 2). It has been confirmed that the GAP domain of p190 RhoGAP possesses GAP activity and that the conserved Lys-1311 (Lys-1223 in RGA-5) residue is essential for this activity (Supplementary Fig. 5b ${ }^{29,30}$. Overexpression of the mutant GAP(K1223A), in which the Lys-1223 in the RGA-5 GAP domain was mutated to alanine, did not cause reduced regeneration (Fig. $4 \mathrm{~d}$ and Supplementary Table 2). These results suggest that the RGA-5 GAP domain stimulates the conversion of RHO-1 to the GDP-bound inactive form, resulting in the inhibition of axon regeneration. In this manner, RGA-5 acts as a potent inhibitor of axon regeneration. We further examined possible genetic interactions between $r g a-5$ and $r h g f-1$, and found that the rga-5(ok2241) mutation was able to suppress the rhgf-1(ok880) defect (Fig. 4d and Supplementary Table 2). This can be explained because RHO-1 can still be weakly activated in the absence of its GEF, thus concomitant loss of GAP affects the phenotype.

How does RHO-1 regulate axon regeneration? Mammalian RhoA has been shown to bind and inhibit the DAG kinase DGK $\theta$ (refs 31,32). DAG kinases remove DAG by phosphorylating the membrane-bound secondary messenger DAG, converting it to phosphatidic acid ${ }^{33,34}$, which implicates RhoA in regulating DAG levels ${ }^{31}$. In addition, C. elegans RHO-1 is able to bind to DGK-1, the C. elegans ortholog of DGK $\theta$ (ref. 35). Thus, DGK-1 is a possible target of negative regulation by $\mathrm{RHO}-1$ (Fig. 3b). If RHO-1 modulates axon regeneration through the inhibition of DGK-1, then we would predict that a $d g k-1$ loss-of-function mutation should suppress the regeneration defect by inactivation of RHO-1. As expected, we found that the $d g k-1$ (ok1462) null mutation suppressed the defect caused by the rhgf-1(ok880) deletion mutation or overexpression of the RGA-5 GAP domain (Fig. 4d and Supplementary Table 2). Our results are most consistent with a signal transduction cascade involving activation of RHO-1, inhibition of DGK-1, and a consequent increase in DAG (Fig. 3b).

SER-7 promotes axon regeneration via the cAMP pathway. If the SER-7-GPA-12 pathway acts upstream of RHO-1 to promote axon regeneration, an $r g a-5$ or $d g k-1$ mutation should suppress the defect in axon regeneration seen in ser-7 and gpa-12 mutants. As expected, the rga-5(ok2241) and $d g k-1$ (ok1462) mutations were each able to suppress the gpa-12 defect (Fig. 5a and Supplementary Table 2). However, in contrast to the gpa-12 mutation, the ser-7 defect was not suppressed by the rga5(ok2241) or $d g k-1$ (ok1462) mutation (Fig. 5a and Supplementary Table 2). These results suggest that another regulatory pathway exists downstream of SER-7, in addition to the GPA-12-RHO-1 pathway, that is required for axon regeneration.

Since the SER-7 5-HT receptor has also been reported to be coupled to GSA-1 Gs $\alpha$ (Fig. 5b) ${ }^{23,36}$, we hypothesized that an additional pathway of SER-7-mediated axon regeneration could involve the GSA-1 signalling pathway. Since Gs $\alpha$ is coupled to adenylyl cyclase, Gs $\alpha$ signalling can be monitored by intracellular cAMP levels. As observed previously ${ }^{37}$, axon regeneration was inhibited by overexpression of $p d e-4$, which encodes a cAMPphosphodiesterase and reduces intracellular cAMP levels (Fig. 5c and Supplementary Table 2). Furthermore, a loss-of-function mutation of $a c y-1$, which encodes adenylyl cyclase, was defective in axon regeneration (Fig. $5 c$ and Supplementary Table 2). These results suggest that activation of GSA-1 signalling is necessary to activate axon regeneration. To confirm that ser-7 and acy-1 function in the same pathway, we constructed $\operatorname{ser}-7(\operatorname{tm} 1325)$; acy-1(nu329) double mutants. The ser-7 mutation did not 
a

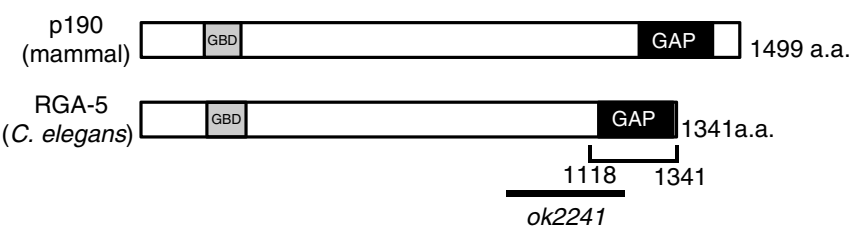

b

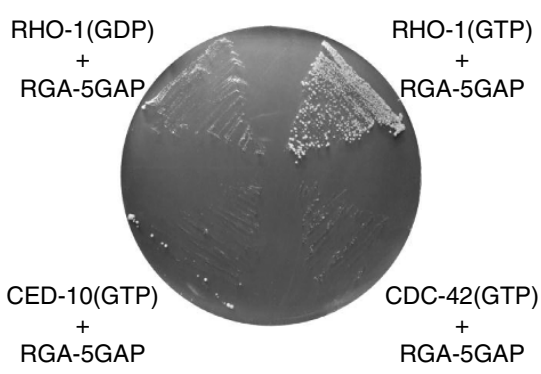

C

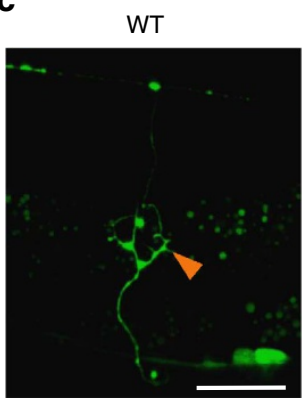

WT +Punc-25::rga-5gap

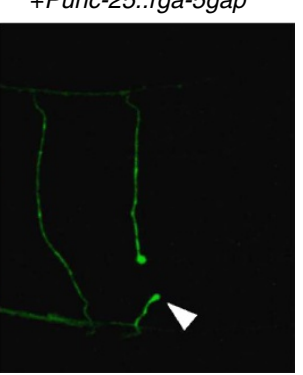

d

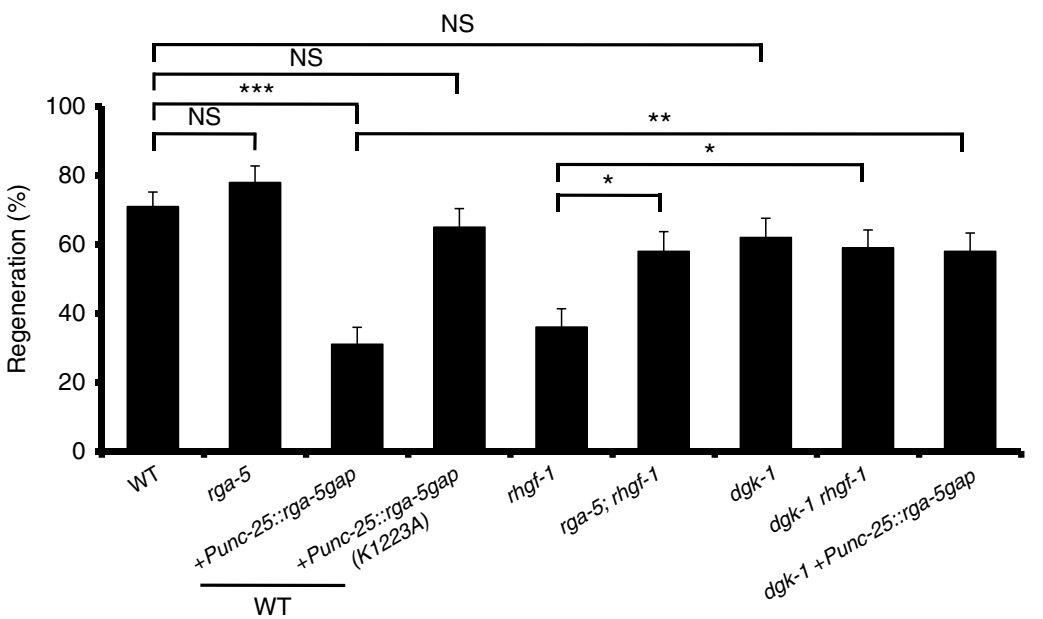

Figure 4 | Effect of RhoGAP on axon regeneration. (a) Structure of RGA-5. Schematic diagrams of RGA-5 and its human counterpart are shown. Domains are shown as follows: a GTP binding domain (GBD) and a Rho GAP domain (GAP). The bold line underneath indicates the extent of the deleted region in the rga-5(ok2241) mutant. (b) A two-hybrid assay for the interaction of RGA-5 with RHO-1. The reporter strain PJ69-4A was co-transformed with expression vectors encoding GAL4 DBD-RHO-1 (T19N) (GDP), GAL4 DBD-RHO-1 (G14V) (GTP), GAL4 DBD-CED-10 (G12V) (GTP), GAL4 DBD-CDC-42 (G12V) (GTP) and GAL4 AD-RGA-5 (GAP domain) as indicated. (c) Representative D-type motor neurons in wild-type animals and wild-type animals overexpressing RGA-5 GAP domain $24 \mathrm{~h}$ after laser surgery. In wild-type animals, severed axons have regenerated growth cones (orange arrowhead). In wild-type animals overexpressing the RGA-5 GAP domain, the proximal end of axon failed to regenerate (white arrowhead). Scale bar $=20 \mu \mathrm{m}$.

(d) Percentages of axons that initiated regeneration $24 \mathrm{~h}$ after laser surgery. Error bars indicate $95 \% \mathrm{Cl}$. ${ }^{\star \star \star} P<0.001$; ${ }^{\star \star} P<0.01$; ${ }^{\star} P<0.05$;

NS, not significant (Fisher's exact test, two-tailed, $n \geq 50$ ). $\mathrm{Cl}$, confidence interval.

enhance the axon regeneration defect of acy-1 mutants (Fig. $5 \mathrm{c}$ and Supplementary Table 2), supporting the possibility that SER-7 functions in the GSA-1 signalling pathway to activate axon regeneration.

Since SER-7 activates both GPA-12 and GSA-1 signalling pathways (Fig. 5b $)^{23}$, we speculated that activation of both pathways are required for axon regeneration. If so, activation of the GSA-1 signalling pathway should suppress the ser-7 defect in axon regeneration in a manner dependent on the activation of the GPA-12 signalling pathway. Treatment of animals with forskolin is expected to cause an increase in cAMP levels by activating adenylyl cyclase ${ }^{37,38}$. We found that forskolin treatment failed to suppress the single mutant ser-7(tm1325) defect in axon regeneration (Fig. 5a and Supplementary Table 2). However, forskolin treatment was able to suppress the axon regeneration defect in ser-7; rga-5 double mutants, in which the GPA-12 signalling pathway is activated (Fig. 5a and Supplementary
Table 2). These results suggest that SER-7 activates GPA-12 and GSA-1, which act in concert to activate axon regeneration.

\section{Discussion}

The neurotransmitter 5-HT plays multiple roles in the enteric, peripheral and central nervous system, and its most prominent biological function is as a messenger transmitting signals from pre- to post-synaptic neurons. However, 5-HT has been reported to play other roles, such as the regulation of neurite growth ${ }^{39,40}$, suggesting that 5 -HT also plays a prominent role in regulating the neuronal cytoarchitecture ${ }^{41,42}$. In C. elegans, 5-HT modulates cognitive behaviours such as chemosensation, learning and memory in addition to a variety of stress responses ${ }^{10,13,16}$. Here our results demonstrate that $C$. elegans 5 -HT is synthesized in axotomized 'non-serotonergic' neurons and is required for axon regeneration. This effect is likely due to a direct action of 5-HT on 


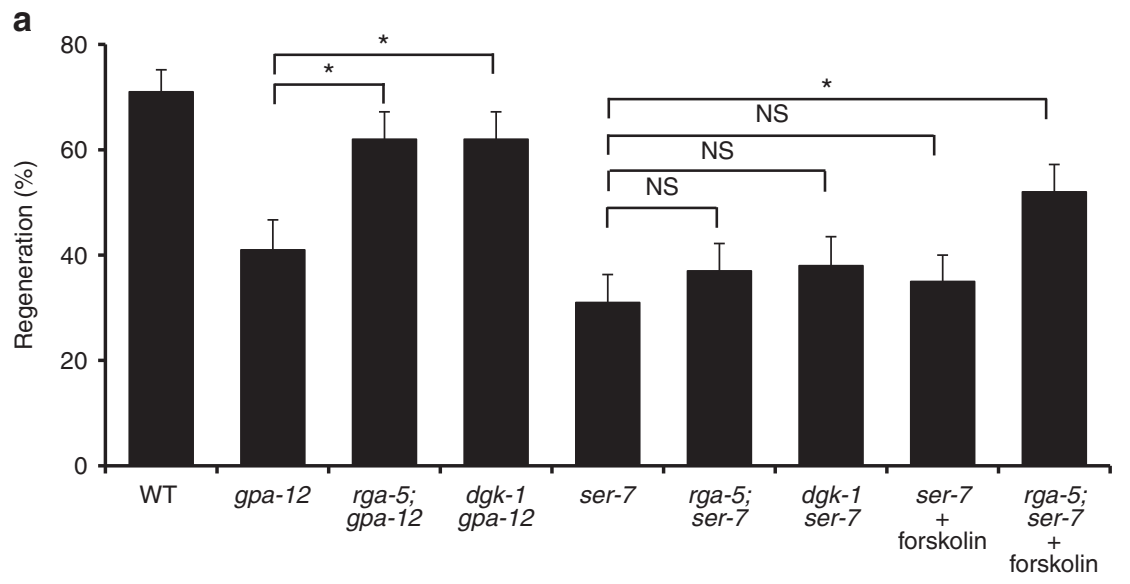

b

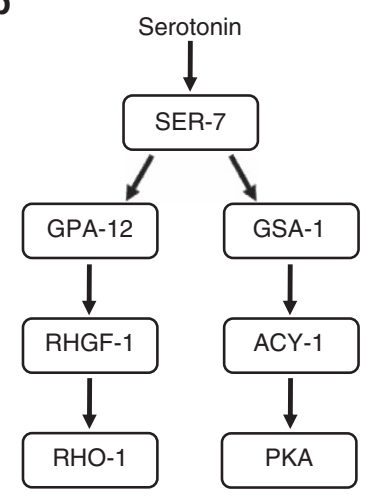

C

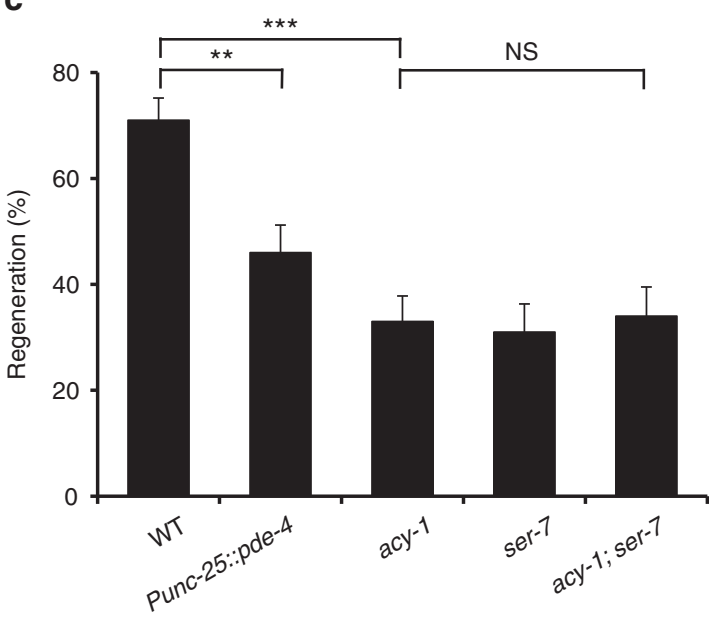

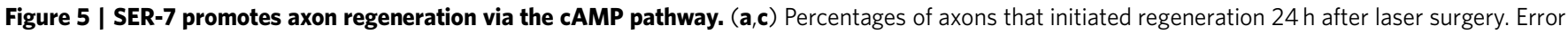
bars indicate $95 \% \mathrm{Cl} .{ }^{\star \star \star} P<0.001 ;{ }^{\star \star} P<0.01 ;{ }^{\star} P<0.05$; NS, not significant (Fisher's exact test, two-tailed, $n \geq 50$ ). (b) SER-7 signalling pathways. SER-7 activates both GPA-12-RHGF-1-RHO-1 and GSA-1-ACY-1-PKA pathways.

injured neurons through the SER-7 5-HT receptor, which activates axon regeneration in a cell-autonomous manner.

Neurons are known to be able to produce distinct sets of neurotransmitters ${ }^{43}$, and some studies have shown that certain stimuli can induce neurotransmitter switching ${ }^{44,45}$. In mammals, leukaemia inhibitory factor and ciliary neurotrophic factor have been shown to induce the differentiation of developing peripheral sympathetic neurons, which normally release noradrenaline, into cholinergic neurons ${ }^{46,47}$. Another study revealed that the balance of the two hippocampal granule cell neurotransmitters, glutamate and GABA, changes during post-embryonic development, as well as with seizures and long-term potentiation ${ }^{48}$. Recently, Dulcis et al. ${ }^{49}$ reported that the neurotransmitters produced by hypothalamic neurons switch between dopamine and somatostatin in response to photoperiod stimuli. Although these studies were largely carried out using populations of neuronal cells that could include different types of cells such as reserve pool neurons, they indicate that neurotransmitter production varies dynamically in neurons. Our findings in C. elegans demonstrate that a single, differentiated GABAergic D-type motor neuron or glutamatergic PLM sensory neuron that is never serotonergic under standard laboratory conditions can be induced to produce 5-HT as a result of axotomy in a manner dependent on HIF-1. This represents a novel case in which a switch in neurotransmitter expression can be regulated. Hypoxia also stimulates expression of 5 -HT via HIF-1 in glutamatergic
ASG neurons ${ }^{16,50}$. However, stabilization of HIF-1, which phenocopies the hypoxia-induced neuronal effects, is sufficient to induce $t p h-1$ expression in ASG neurons, but not in D or PLM neurons. Therefore, HIF-1-dependent $t p h-1$ expression in severed neurons is regulated by a mechanism different from the HIF-1stabilizing mechanism.

In mammals, the expression and stability of HIF-1, as well as the expression of its target genes, are all increased after spinal cord injury ${ }^{51,52}$. Thus, activation of HIF-1 by axotomy may be conserved among animals. Furthermore, the HIF-induced upregulation of $5-\mathrm{HT}$ in specific regions of the vertebrate brain $^{53}$ suggests that the mechanisms coupling axon regeneration and 5-HT signalling to produce an alteration in neuronal responses may be conserved among animals. Therefore, it would be interesting to know whether axotomy of vertebrate neurons also stimulates expression of 5-HT or other neurotransmitters that influence regeneration. The inability of axons to regenerate in the adult mammalian central nervous system is a major problem in a number of diseases, and understanding the multi-functional roles of 5-HT signalling on neurite growth may lead to the development of novel neuroregenerative therapies by targeting serotonin-regulated signalling pathways.

The biological effects of 5-HT are mediated through interactions with $G$ protein-coupled receptors ${ }^{22}$. Here we have shown that the activated SER-7 5-HT receptor promotes axon 
regeneration in injured neurons by activating G12 $\alpha$ and Gs $\alpha$ signalling pathways in a cell-autonomous manner. How do G12 $\alpha$ and Gs $\alpha$ signalling cascades activate axon regeneration? Several studies suggested that DAG and cAMP act downstream of the G1 $2 \alpha$ and Gs $\alpha$ cascades, respectively ${ }^{54-57}$. In mammals, G12 $\alpha$ activates an RGS-containing RhoGEF, which in turn activates RhoA. Several lines of evidence suggest that this pathway also acts within C. elegans motor neurons ${ }^{26}$. The axon regeneration pathway is regulated by both of these components acting in the G12 $\alpha$ pathway, as either a mutation in rhgf- 1 encoding the RGS-containing RhoGEF or inactivation of RHO-1 by RhoGAP RGA-5 inhibits axon regeneration. In addition, RHO- 1 activation is able to suppress the defect in axon regeneration caused by the gpa-12 mutation. Thus, the genetic data support a model in which the G12 $\alpha$-RhoGEF-RhoA signalling pathway is conserved in the control of axon regeneration in C. elegans. Consistent with this, GPA-12 and RHGF-1 are both expressed in motor neurons and physically interact ${ }^{25}$. In C. elegans, activated RHO-1 causes an increase in DAG by directly binding to and inhibiting DGK-1 (ref. 35). High levels of DAG activate the PKC homolog TPA-1, leading to activation of the JNK pathway by phosphorylation of MLK-1 MAPKKK ${ }^{58}$. These results raise the possibility that the G12 $\alpha$ pathway activates axon regeneration by inducing an increase in DAG, resulting in the activation of the JNK pathway.

The Gs $\alpha$ cascade involves the activation of adenylyl cyclase by Gs $\alpha$, and the consequent increase in cAMP production leads to the activation of PKA. Our recent results suggest that in response to axon injury, PKA upregulates transcription of the svh-2 gene, which encodes a growth factor receptor functioning in the JNK pathway 59,60 . Thus, one effector of the Gs $\alpha$ pathway that stimulates axon regeneration is a transcription factor that activates transcription of the $s v h-2$ gene, resulting in activation of the JNK pathway. However, the Gs $\alpha$ pathway likely has additional targets. Taken together, these results suggest that the G12 $\alpha$ and Gs $\alpha$ pathways converge to activate the JNK pathway in axon regeneration.

\section{Methods}

C. elegans strains. C. elegans strains used in this study are listed in Supplementary Table 3. All strains were maintained on nematode growth medium (NGM) plates and fed with bacteria of the OP50 strain, as described previously ${ }^{61}$.

Plasmids. Punc-25::tph-1 was made by inserting the $t p h-1$ cDNA isolated from a cDNA library by PCR into the pSC325 vector. Psrh-142::tph-1 was made by replacing an NLS-Cre cDNA fragment of Psrh-142::nls-cre plasmid ${ }^{11}$ (Gift from Dr Cori Bargmann) with a tph-1 cDNA fragment. Punc-25::nls-cre was generated by inserting NLS-Cre cDNA (Addgene) into the pSC325 vector. Punc-47::mcherry was generated by replacing the $g f p$ DNA of the pOKU64 vector (Punc-47::gfp, a gift from Dr Ikue Mori) with the mCherry cDNA (DNA2.0). Punc-25::ser-7 and Pmec-7::ser-7 were made by inserting the ser-7 cDNA isolated from a cDNA library by PCR into the pSC325 and pPD52.102 vectors, respectively. Punc-25::rho-1 was generated by inserting the rho-1 cDNA, provided from Dr Kozo Kaibuchi (Nagoya University, JAPAN), into the pSC325 vector. Punc-25::rho-1 (G14V) and Punc-25::rho-1 (T19N) were made by oligonucleotide-directed PCR using Punc-25::rho-1 as a template and the mutations were verified by DNA sequencing. The Punc-25::rga-5gap plasmid contains the GAP domain of rga-5 cDNA isolated from a cDNA library by PCR using oligonucleotides $5^{\prime}$-gtcagcATGAAGAA CAAAGTGCAACTCATGATC- $3^{\prime}$ and $5^{\prime}$-ccatGGCCCGATAATTATCCACG TCATAG- $3^{\prime}$, inserted into the pSC325 vector. The Punc-25::rga-5gap (K1223A) was made by oligonucleotide-directed PCR using Punc-25::rga-5gap as a template and the mutation was verified by DNA sequencing. Punc-25::pde-4 was generated by inserting the pde-4 cDNA isolated from a cDNA library into the pSC325 vector. The GAL4 DBD-RHO-1 (G14V), GAL4 DBD-RHO-1 (T19N), GAL4 DBD-CED-10 (G12V) and GAL4 DBD-CDC-42 (G12V) plasmids are also generous gifts from Dr Kozo Kaibuchi. The GAL4 AD-RGA-5 (GAP domain) plasmid was constructed by inserting the rga-5gap cDNA into the pACTII vector. The Pmyo-2::dsred-monomer plasmid has been described previously ${ }^{59}$.

Transgenic animals. Transgenic animals were obtained as described in ref. 62 . Punc-25::rga-5gap (50 ng $\left.\mathrm{l}^{-1}\right)$, Punc-25::rga-5gap(K1223A) $\left(50 \mathrm{ng}^{-1}{ }^{-1}\right)$, Punc-25::rho-1 (G14V) (5 ng $\left.\mu \mathrm{l}^{-1}\right)$, Punc-25::rho-1 (T19N) (5 ng $\left.\mu \mathrm{l}^{-1}\right)$,
Punc-25::ser-7 (50 ng $\left.\mathrm{ll}^{-1}\right)$, Pmec-7::ser-7 (5 ng $\left.\mathrm{l}^{-1}\right)$, Punc-25::pde-4 (50 ng $\left.\mu^{-1}\right)$, Punc-25::tph-1 (50 ng $\left.\mu \mathrm{l}^{-1}\right)$, Psrh-142::tph-1 (50 ng $\mu \mathrm{l}^{-1}$ ), Punc-25::nls-cre (50 ng $\left.\mu^{-1}\right)$ and Punc-47::mcherry (100 ng $\left.\mu \mathrm{l}^{-1}\right)$ plasmids were used to generate kmEx1301 [Punc-25::rga-5gap + Pmyo-2::dsred-monomer], kmEx1312 [Punc-25::rga-5gap(K1223A) + Pmyo-2::dsred-monomer], kmEx1302 [Punc-25::rho-1 (G14V) + Pmyo-2::dsred-monomer], kmEx1303 [Punc-25::rho-1 $($ T19N $)+$ Pmyo-2::dsred-monomer], kmEx1304 [Punc-25::ser-7 + Pmyo-2::dsredmonomer], kmEx1305 [Pmec-7::ser-7 + Pmyo-2::dsred-monomer], kmEx1306 [Punc-25::pde-4 + Pmyo-2::dsred-monomer], kmEx1307 [Punc-25::tph-1+ Pmyo-2::dsred-monomer], kmEx1311 [Psrh-142::tph-1 + Pmyo-2::dsred-monomer], kmEx1308 [Punc-25::nls-cre + Pmyo-2::dsred-monomer] and kmEx1309 [Punc-47::mcherry + Pmyo-2::dsred-monomer], respectively. The kyEx4057 [Pceh-2::nls-cre], kyEx4077 [Psrh-142::nls-cre], kyEx4107 [Pegl-6::nls-cre] and kySi56 [floxed tph-1 genomic rescue] were generous gifts from Dr Cori Bargmann $^{11}$. The otIs 197, zdIs13, wpIs36 and wdEx848 transgenes ${ }^{63-65}$ were obtained from CGC.

Yeast two-hybrid assay. GAL4 DBD-RHO-1(G14V, T19N), GAL4 DBD-CED-10 (G12V), GAL4 DBD-CDC-42 (G12V) and GAL4 AD-RGA-5 (GAP domain) plasmids were co-transformed into the Saccharomyces cerevisiae reporter strain PJ69-4A and allowed to grow on SC-Leu-Trp plates. Transformants grown on these plates were then streaked out onto SC-Leu-Trp-His plates and incubated at $30^{\circ} \mathrm{C}$ for 4 days.

Axotomy. All animals were subjected to axotomy at the young adult stage at $20^{\circ} \mathrm{C}$ Young adult hermaphrodites were immobilized with $20 \mathrm{mM}$ sodium azide in M9 buffer on $2 \%$ agarose pad under a cover slip. D-type motor neurons expressing GFP were imaged with a fluorescence microscope. Selected commissural axons, mainly posterior D-type neurons, were cut using a 440-nm MicroPoint ablation Laser System from Photonic Instruments. After surgery, animals were allowed to recover on an agar plate and remounted for fluorescent imaging $\sim 24 \mathrm{~h}$ after surgery. For measurements of D neurons, imaged commissures that exhibited growth cones or small branches on the proximal fragment were counted as 'regenerated'. The proximal fragments that showed no change after $24 \mathrm{~h}$ were counted as 'no regeneration'. A minimum of 50 individuals with 1-3 axotomized commissures were observed for most experiments. For PLM touch neurons, extended process lengths were measured from maximum transparency projections of a single $\mathrm{Z}$-stack, by using the Keyence BZ-X700 analysis software.

Drug experiments. 5-HT and forskolin were obtained from Wako and Focus Biomolecules, respectively. For 5-HT treatment, worms were grown from egg to adult, up until axotomy, on NGM plates containing $6.5 \mathrm{mM} \mathrm{5-HT}$ and OP50 Escherichia coli. After axotomy, the worms were plated on NGM plates containing 5-HT and OP50 E. coli for 24 h. For forskolin treatment, the mixed stages of worms were incubated with M9 buffer containing $100 \mu \mathrm{m}$ forskolin and heat-killed OP50 for $12 \mathrm{~h}$ at $20^{\circ} \mathrm{C}$, and the worms at young adult stage after the treatment were used.

Immunofluorescence. 5-HT immunofluorescence was essentially performed as described previously ${ }^{5}$. Briefly, the worms axotomized either D neurons or PLM neurons were fixed by $4 \%$ formaldehyde in PBS buffer at $4{ }^{\circ} \mathrm{C}$ for overnight, washed three times by PBS and then treated by PBS containing $5 \%$ $\beta$-mercaptoethanol and $1 \%$ Triton X-100 for $24 \mathrm{~h}$. Then, the worms were washed by PBS and digested in $100 \mathrm{mM}$ Tris- $\mathrm{HCl}$ (pH7.5), $1 \mathrm{mM} \mathrm{CaCl}_{2}$ and $5 \mathrm{mg} \mathrm{ml}^{-1}$ collagenase (Wako, 034-10533). After digestion, a rat anti-serotonin antibody (Chemicon, Cat. No. MAB352), 100X diluted with Can Get Signal Immunostain (TOYOBO), was used as the first antibody solution. A chicken anti-rat Alexa 594 (Molecular probes), 200X diluted with Can Get Signal Immunostain (TOYOBO), was used as the second antibody solution.

Microscopy. Standard fluorescence images of transgenic worms were observed and photographed, using either a Zeiss Plan-APOCHROMAT $\times 100$ objective of a Zeiss Axioplan II fluorescence microscope with a Hamamatsu 3CCD camera, or a $\times 100$ objective of a Keyence BZ-X700 all-in-one fluorescence microscope Confocal fluorescence images were taken on an Olympus FV500 and FV1000 confocal laser scanning microscopes with $\times 60$ and $\times 100$ objectives.

Quantification of GFP expression. Expression of GFP fluorescence was quantified using the ImageJ program (NIH). The cell bodies of severed D or PLM neurons were outlined with closed polygons and the fluorescence intensities within these areas were determined $\left(I_{\mathrm{s}}\right)$. Then $I_{\mathrm{s}}$ was divided by the area to derive intensity per area $\left(I_{\mathrm{sa}}\right)$. To determine the background intensity of each cell, the same polygon was placed in an area neighbouring the cell body, fluorescence was measured $\left(I_{\mathrm{b}}\right)$, and this was divided by area $\left(I_{\mathrm{ba}}\right)$. The relative signal intensity per area $\left(I_{\mathrm{ra}}\right)$ was calculated as $I_{\mathrm{sa}}-I_{\mathrm{ba}}$. Cells having $I_{\mathrm{ra}}>7$ (for D neuron) or $I_{\mathrm{ra}}>4$ (for PLM neuron) were categorized as 'expressed', respectively. 
Statistical analysis. Statistical analyses were carried out as described previously ${ }^{58}$. Briefly, confidence intervals (95\%) were calculated by the modified Wald method and two-tailed $P$ values were calculated using Fisher's exact test (http://www.graphpad.com/quickcalcs/). The Welch's $t$-test was performed by using a $t$-test calculator (http://www.graphpad.com/quickcalcs/ttest1/).

\section{References}

1. Yanik, M. F. et al. Neurosurgery: functional regeneration after laser axotomy. Nature 432, 822 (2004)

2. Hammarlund, M. \& Jin, Y. Axon regeneration in C. elegans. Curr. Opin. Neurobiol. 27, 199-207 (2014)

3. Chen, L. et al. Axon regeneration pathways identified by systematic genetic screening in C. elegans. Neuron 71, 1043-1057 (2011).

4. Nix, P. et al. Axon regeneration genes identified by RNAi screening in C. elegans. J. Neurosci. 34, 629-645 (2014)

5. Sze, J. Y., Victor, M., Loer, C., Shi, Y. \& Ruvkun, G. Food and metabolic signalling defects in a Caenorhabditis elegans serotonin-synthesis mutant. Nature 403, 560-564 (2000).

6. de Kloet, E. R., Joels, M. \& Holsboer, F. Stress and the brain: from adaptation to disease. Nat. Rev. Neurosci. 6, 463-475 (2005).

7. McEwen, B. S. Physiology and neurobiology of stress and adaptation: central role of the brain. Physiol. Rev. 87, 873-904 (2007).

8. Joels, M. \& Baram, T. Z. The neuro-symphony of stress. Nat. Rev. Neurosci. 10, 459-466 (2009)

9. Chang, A. J., Chronis, D. S., Karow, D. S., Marletta, M. A. \& Bargmann, C. I. A distributed chemosensory circuit for oxygen preference in C. elegans. PLoS Biol. 4, e274 (2006)

10. Liang, B., Moussaif, M., Kuan, C. J., Gargus, J. J. \& Sze, J. Y. Serotonin targets the DAF-16/FOXO signaling pathway to modulate stress responses. Cell Metab. 4, 429-440 (2006).

11. Flavell, S. W. et al. Serotonin and the neuropeptide PDF initiate and extend opposing behavioral states in C. elegans. Cell 154, 1023-1035 (2013).

12. Rand, J. B. \& Nonet, M. L. Synaptic transmission in C. elegans II. (eds Riddle, D. L. et al.) 611-643 (Cold spring Harbor Laboratory Press, 1997).

13. Chase, D. L. \& Koelle, M. R. Biogenic amine neurotransmitters in C. elegans. The C. elegans research community, WormBook, doi/10.1895/wormbook. 1.132.1, http://www.wormbook.org (2007).

14. Jorgensen, E. M. GABA. The C. elegans Research Community, WormBook, doi/10.1895/wormbook.1.14.1, http://www.wormbook.org (2005).

15. Lee, R. Y. N., Sawin, E. R., Chalfie, M., Horvitz, H. R. \& Avery, L. EAT-4, a homolog of a mammalian sodium-dependent inorganic phosphate cotransporter, is necessary for glutamatergic neurotransmission in Caenorhabditis elegans. J. Neurosci. 19, 159-167 (1999).

16. Pocock, R. \& Hobert, O. Hypoxia activates a latent circuit for processing gustatory information in C. elegans. Nat. Neurosci. 13, 610-614 (2010).

17. Jiang, H., Guo, R. \& Powell-Coffman, J. A. The Caenorhabditis elegans hif-1 gene encodes a bHLH-PAS protein that is required for adaptation to hypoxia. Proc. Natl Acad. Sci. USA 98, 7916-7921 (2001).

18. Epstein, A. C. et al. C. elegans EGL-9 and mammalian homologs define a family of dioxygenases that regulate HIF by prolyl hydroxylation. Cell 107, 43-54 (2001).

19. Bishop, T. et al. Genetic analysis of pathways regulated by the von HippelLindau tumor suppressor in Caenorhabditis elegans. PLoS Biol. 2, e289 (2004).

20. Shen, C., Nettleton, D., Jiang, M., Kim, S. K. \& Powell-Coffman, J. A. Roles of the HIF-1 hypoxia-inducible factor during hypoxia response in Caenorhabditis elegans. J. Biol. Chem. 280, 20580-20588 (2005).

21. Pocock, R. \& Hobert, O. Oxygen levels affect axon guidance and neuronal migration in Caenorhabditis elegans. Nat. Neurosci. 11, 894-900 (2008).

22. Hoyer, D., Hannon, J. P. \& Martin, G. R. Molecular, pharmacylogical and functional diversity of 5-HT receptors. Pharmacol. Biochem. Behav. 71, 533-554 (2002)

23. Song, B. \& Avery, L. Serotonin activates overall feeding by activating two separate neural pathways in Caenorhabditis elegans. J. Neurosci. 32, 1920-1931 (2012).

24. Suzuki, N., Nakamura, S., Mano, H. \& Kozasa, T. G $\alpha 12$ activates Rho GTPase through tyrosine-phosphorylated leukemia-associated RhoGEF. Proc. Natl Acad. Sci. USA 100, 733-738 (2003).

25. Yau, D. M. et al. Identification and molecular characterization of the G $\alpha 12-\mathrm{Rho}$ guanine nucleotide exchange factor pathway in Caenorhabditis elegans. Proc. Natl Acad. Sci. USA 100, 14748-14753 (2003).

26. Hiley, E., McMullan, R. \& Nurrish, S. J. The G $\alpha 12-$ RGS RhoGEF-RhoA signalling pathway regulates neuromodulator release in C. elegans. $E M B O J .25$, 5884-5895 (2006).

27. Bos, J. L., Rehmann, H. \& Wittingghofer, A. GEFs and GAPs: critical elements in the control of small G proteins. Cell 129, 865-877 (2007).

28. Hammarlund, M., Nix, P., Hauth, L., Jorgensen, E. M. \& Bastiani, M. Axon regeneration requires a conserved MAP kinase pathway. Science 323, 802-806 (2009).
29. Ridley, A. J. et al. Rho family GTPase activating proteins p190, bcr and rhoGAP show distinct specificities in vitro and in vivo. EMBO J. 12, 5151-5160 (1993).

30. Wolf, R. M. et al. p190RhoGAP can act to inhibit PDGF-induced gliomas in mice: a putative tumor suppressor encoded on human chromosome $19 q 13.3$. Genes Dev. 17, 476-487 (2003).

31. Houssa, B., de Widt, J., Kranenburg, O., Moolenaar, W. H. \& van Blitterswijk, W. J. Diacylglycerol kinase $\theta$ binds to and is negatively regulated by active RhoA. J. Biol. Chem. 274, 6820-6822 (1999).

32. Los, A. P., Vinke, F. P., de Widt, J., Divecha, N. \& van Blitterswijk, W. J. Structure-activity relationship of diacylglycerol kinase $\theta$. Biochim. Biophys Acta. 1636, 169-174 (2004).

33. van Blitterswijk, W. J. \& Houssa, B. Properties and functions of diacylglycerol kinase. Cell Signal. 12, 595-605 (2000).

34. Luo, B., Prescott, S. M. \& Topham, M. K. Diacylglycerol kinase $\zeta$ regulates phosphatidylinositol 4-phosphate 5-kinase I $\alpha$ by a novel mechanism. Cell Signal. 16, 891-897 (2004).

35. McMullan, R., Hiley, E., Morrison, P. \& Nurrish, S. J. Rho is a presynaptic activator of neuromodulator release at pre-existing synapses in C. elegans. Gene Dev 20, 65-76 (2005).

36. Hobson, R. J., Geng, J., Gray, A. D. \& Komuniecki, R. W. SER-7b, a constitutively active $\mathrm{G} \alpha$ s coupled $5-\mathrm{HT}_{7}$-like receptor expressed in the Caenorhabditis elegans M4 pharyngeal motorneuron. J. Neurochem. 87, 22-29 (2003).

37. Ghosh-Roy, A., Wu, Z., Goncharov, A., Jin, Y. \& Chisholm, A. D. Calcium and cyclic AMP promote axonal regeneration in Caenorhabditis elegans and require DLK-1 kinase. J. Neurosci. 30, 3175-3183 (2010).

38. Seamon, K. B., Padgett, W. \& Daly, J. W. Forskolin: unique diterpene activator of adenylate cyclase in membranes and in intact cells. Proc. Natl Acad. Sci. USA 78, 3363-3367 (1981).

39. Trakhtenberg, E. F. \& Goldberg, J. L. The role of serotonin in axon and dendrite growth. Int. Rev. Neurobiol. 106, 105-126 (2012)

40. Rojas, P. S., Neira, D., Muñoz, M., Lavandero, S. \& Fielder, J. L. Serotonin (5-HT) regulates neurite outgrowth through 5-HT1A and 5-HT7 receptors in cultured hippocampal neurons. J. Neurosci. Res. 92, 1000-1009 (2014).

41. Hydon, P. G., McCobb, D. P. \& Kater, S. B. Serotonin selectively inhibits growth cone motility and synaptogenesis of specific identified neurons. Science 226, 561-564 (1984).

42. Kvachnina, E. et al. 5-HT7 receptor is coupled to G $\alpha$ subunits of heterotrimeric G12-protein to regulate gene transcription and neuronal morphology. J. Neurosci. 25, 7821-7830 (2005).

43. Burnstock, G. Cotransmission. Curr. Opin. Phamacol 4, 47-52 (2004).

44. Potter, D. D., Landis, S. C., Matsumoto, S. G. \& Furshpan, E. J. Synaptic functions in rat sympathetic neurons in microcultures. II. Adrenergic/ cholinergic dual status and plasticity. J. Neurosci. 6, 1080-1098 (1986).

45. Spitzer, N. C. Neurotransmitter switching? No surprise. Neuron 86, 1131-1144 (2015).

46. Saadat, S., Sendtner, M. \& Rohrer, H. Ciliary neurotrophic factor induces cholinergic differentiation of rat sympathetic neurons in culture. J. Cell Biol. 108, 1807-1816 (1989).

47. Yamamori, T. et al. The cholinergic neuronal differentiation factor from heart cells is identical to leukemia inhibitory factor. Science 246, 1412-1416 (1989).

48. Gutiérrez, R. The dual glutamatergic-GABAergic phenotype of hippocampal granule cells. Trends Neurosci. 28, 297-303 (2005).

49. Dulcis, D., Jamshidi, P., Leutgeb, S. \& Spitzer, N. C. Neurotransmitter switching in the adult brain regulates behavior. Science 340, 449-453 (2013).

50. Serrano-Saiz, E. et al. Modular control of glutamatergic neuronal identity in $C$. elegans by distinct homeodomain proteins. Cell 155, 659-673 (2013).

51. Ju, Y., He, M. \& Mao, B. Sequential changes of hypoxia-inducible factor $1 \alpha$ in experimental spinal cord injury and its significance. Clin. J. Traumatol 5, 103-106 (2002)

52. Xiaowei, H., Ninghui, Z., Wei, X., Yiping, T. \& Linfeng, X. The experimental study of hypoxia-inducible factor- $1 \alpha$ and its target genes in spinal cord injury. Spinal Cord 44, 35-43 (2006).

53. Poncet, L., Denoroy, L., Daimaz, Y. \& Pequignot, J. M. Activity of tryptophan hydroxylase and content of indolamines in discrete brain regions after a long-term hypoxic exposure in the rat. Brain Res. 765, 122-128 (1997).

54. Berger, A. J., Hart, A. C. \& Kaplan, J. M. G $\alpha$ s-induced neurodegeneration in Caenorhabditis elegans. J. Neurosci. 18, 2871-2880 (1998)

55. Korwagen, H. C., van der Linden, A. M. \& Plasterk, R. H. A. G protein hyperactivation of the Caenorhabditis elegans adenylyl cyclase SGS-1 induces neuronal degeneration. EMBO J. 17, 5059-5065 (1998).

56. van der Linden, A. M., Moorman, C., Cuppen, E., Korwagen, H. C. \& Plasterk, R. H. A. Hyperactivation of the G12-mediated signaling pathway in Caenorhabditis elegans induces a developmental growth arrest via protein kinase C. Curr. Biol. 13, 516-521 (2003).

57. Schade, M. A., Reynolds, N. K., Dollins, C. M. \& Miller, K. G. Mutations that rescue the paralysis of Caenorhabditis elegans ric-8 (synembryn) mutants 
activate the Gas pathway and define a third major branch of the synaptic signaling network. Genetics 169, 631-649 (2005).

58. Pastuhov, S. I. et al. Endocannabinoid-Go $\alpha$ signalling inhibits axon regeneration in Caenorhabditis elegans by antagonizing Gq $\alpha$-PKC-JNK signalling. Nat. Commun. 3, e1136 (2012).

59. Li, C. et al. The growth factor SVH-1 regulates axon regeneration in C. elegans via the JNK MAPK cascade. Nat. Neurosci. 15, 551-557 (2012).

60. Li, C., Hisamoto, N. \& Matsumoto, K. Axon regeneration is regulated by Ets C/EBP transcription complexes generated by activation of the cAMP/Ca2+ signaling pathways. PLoS Genet. 11, e1005603 (2015).

61. Brenner, S. The genetics of Caenorhabditis elegans. Genetics 77, 71-94 (1974).

62. Mello, C. C., Kramer, J. M., Stinchcomb, D. \& Ambros, V. Efficient gene transfer in C. elegans: extrachromosomal maintenance and integration of transforming sequences. EMBO J. 10, 3959-3970 (1991).

63. Clark, S. G. \& Chiu, C. C. elegans ZAG-1, a Zn-finger-homeodomain protein, regulates axonal development and neuronal differentiation. Development 130, 3781-3794 (2003).

64. Firnhaber, C. \& Hammarlund, M. Neuron-specific RNAi in C. elegans and its use in a screen for essential genes required for GABA neuron function. PLoS Genet. 9, e1003921 (2013).

65. Smith, C. J. et al. Sensory neuron fates are distinguished by a transcriptional switch that regulates dendrite branch stabilization. Neuron 79, 266-280 (2013).

\section{Acknowledgements}

We thank Dr Cori Bargmann, Dr Kozo Kaibuchi, Dr Ikue Mori, Caenorhabditis Genetic Center (CGC), National Bio-Resource Project and C. elegans Knockout Consortium for materials. Some strains were provided by the CGC, which is funded by NIH Office of Research Infrastructure Programs (P40 OD10440). This work was supported by grants from the Ministry of Education, Culture and Science of Japan (to K.M. and N.H.), as well as from the Naito Foundation (to N.H.).

\section{Author contributions}

T.A., H.M., N.H. and K.M. designed the experiments and analyzed data. T.A., H.M., C.L and S.P. performed genetic and biological experiments. P.N. and M.B. identified rga-5 and shared the information. T.A. and H.M. performed all of the other experiments. N.H and K.M. wrote the manuscript.

\section{Additional information}

Supplementary Information accompanies this paper at http://www.nature.com/ naturecommunications

Competing financial interests: The authors declare no competing financial interests.

Reprints and permission information is available online at http://npg.nature.com/ reprintsandpermissions/

How to cite this article: Alam, T. et al. Axotomy-induced HIF-serotonin signalling axis promotes axon regeneration in C. elegans. Nat. Commun. 7:10388 doi: $10.1038 /$ ncomms10388 (2016).

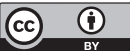

This work is licensed under a Creative Commons Attribution 4.0 International License. The images or other third party material in this article are included in the article's Creative Commons license, unless indicated otherwise in the credit line; if the material is not included under the Creative Commons license, users will need to obtain permission from the license holder to reproduce the material. To view a copy of this license, visit http://creativecommons.org/licenses/by/4.0/ 